\title{
Automatic Chat Generation of Emotional Entertainment Characters Using News Information
}

\author{
Jun'ichi Hoshino, Tetsuya Saito, and Kenichi Hirota \\ University of Tsukuba, Graduate school of Systems and Information Engineering, \\ 1-1-1, Tennodai, Tsukuba-shi, Ibaraki, Japan \\ \{jhoshino, stetsuya, hirota\} @entcomp.esys.tsukuba.ac.jp
}

\begin{abstract}
Currently interactive entertainment characters have their conversation topics prepared beforehand by the creator. In this paper, we propose the automatic chat generation engine for emotional entertainment characters using real-time news information. The character emotionally reacts to the news contents based on their interest and feelings so that the users can have intimate feeling. The character also incrementally learns user's interest from their response.
\end{abstract}

Keywords: chat generation, RSS, emotional character, user learning.

\section{Introduction}

Currently, various games and movies offer the means to conduct conversations with characters. In role-play games, there are often scenes in which a player talks about the progression of the game with non-player characters, hears two characters talking, or joins in the small talk. In this paper, we propose an entertainment character that generates various utterance topics using dynamic sources of news, weather reports, etc., which exist on the Web as sources of real-world information. Since the Web information used is up-to-date, the utterance content of the character can be updated at any time without the need for the creator's intervention. Moreover, an expression of feeling for the topic is a critical factor in improving the expressivity of the utterance, and in this paper, we propose a technique for simply generating the expression of the character's feeling from the directionality of the chat content. Using this technique, the character can express surface feelings appropriate to the topic. The latest news, weather forecasts, etc., are used as chat topics so that generating an appropriate conversation becomes possible using information acquired from the Web.

\section{Related Works}

Studies dealing with a dynamic source involve a sport system based on ball movement, game data, and the team impression in soccer simulation [1]. However, the focus is on an ongoing soccer game, and therefore, not suitable for utterances in chat communications. In addition, studies involving characters that read Web content, convert it into broadcasts and talk-show type content using character animation 
[2][3][4]. Interfaces that interactively retrieve a formula from the user and some characters have also been proposed [5][6].

\section{System Design}

Fig. 1 shows the outline chart of the technique. This system talks about the news and weather as real-world information. The topic is acquired and accumulated from XML and RDF files describing the news, weather forecasts, etc., on the Web, which are delivered as RSS feeds. Hereafter, these data files have been designated Web information files.

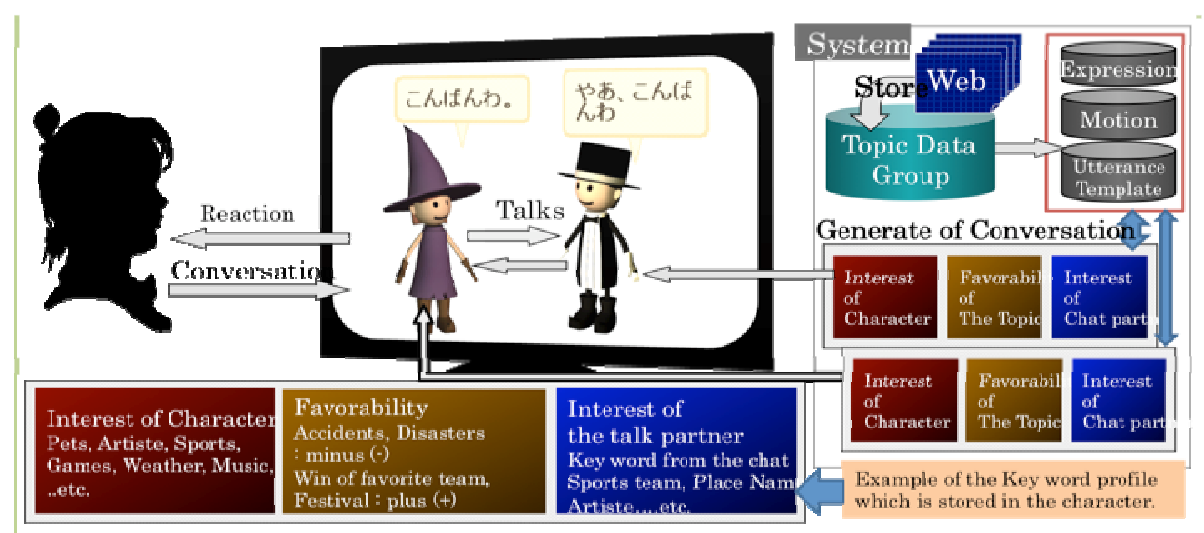

Fig. 1. Outline of the chat generation system

Conversation between the user and the character starts with text that the user inputs or with the character's voluntary utterances to the user. The topic is decided using the user's and character's level of interest to generate a significant utterance for the user, and not just make random conversation. If a topic garners a high level of interest, it can easily be selected as the conversation content. Moreover, the level of interest in the topic changes tending to favor the user and the character through small talk.

This system uses parameters of favorability that judge the topics as pleasant or unpleasant to improve the character's utterances. When the character utters, it automatically calculates the favorability of the topic and selects an appropriate expression, behavior, and the utterance template.

The conversation flow is coordinated according to several patterns and used to enable continuous utterances and an easy conversation between the user and the character. Moreover, the flow of an appropriate conversation between characters is achieved by equipping the character with a conversation pattern that has a level of interest similar to that of the user.

When the conversation begins from the user's text input, the character selects a topic suitable for the user's demand. The input content is accumulated as an information genre in line with the user's interest. When the conversation begins from voluntary utterances made by the character, the topic is selected from the genre in which the character has an interest or that in which the user has an interest. Next, expressions, 
gestures, and the utterance template of the character that match the favorability are selected by referring to the calculated favorability of each topic resulting in an animated utterance.

Fig. 2 shows an example of an expression and operating the character according to favorability. Since these operations consider only the directionality of the content in the chatting topic, the character does not develop feelings that the person actually has beforehand. However, we consider that it is superficially possible to react appropriately for the topic.

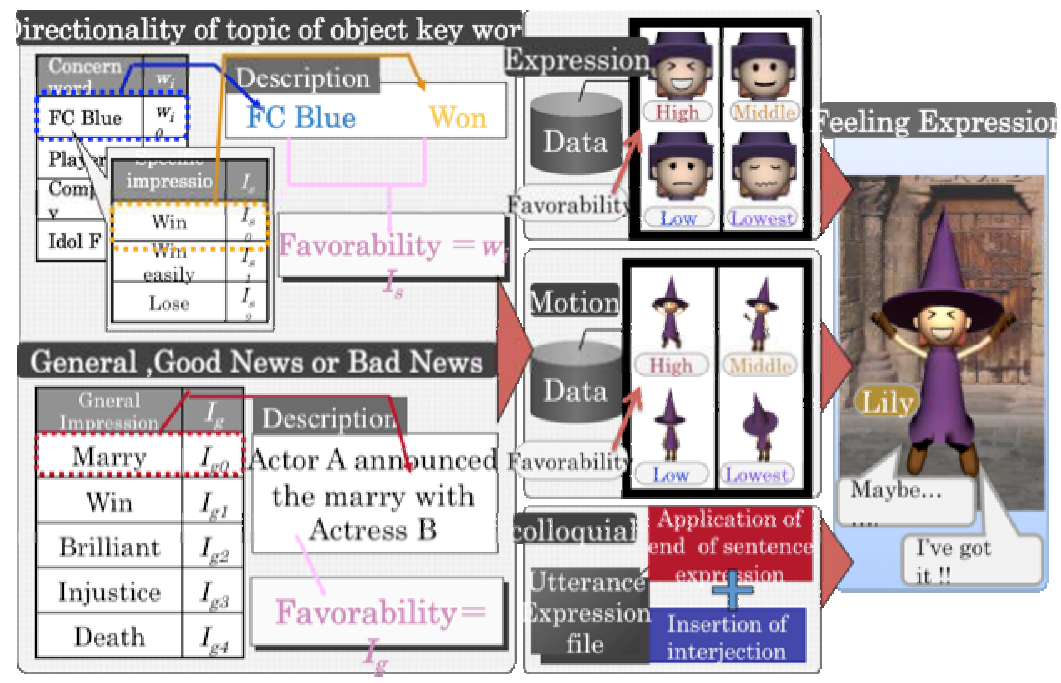

Fig. 2. Example of Feelings and Gestures for the Topic

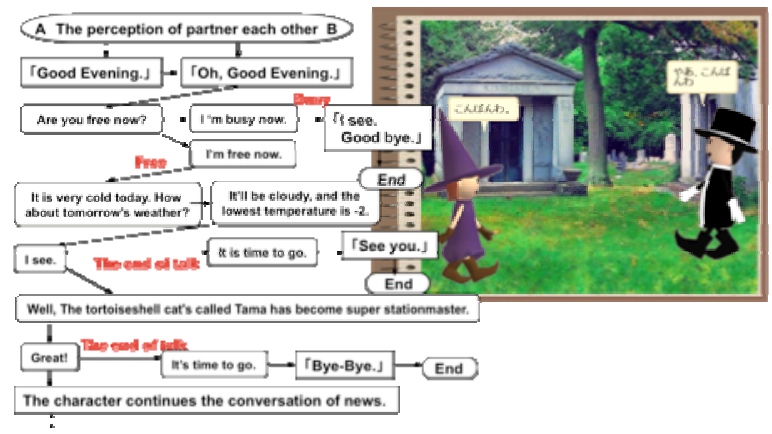

Fig. 3. Example of Conversation between Characters

\section{Conclusion}

In this paper, we propose the concept of emotional chat for characters in games through the use of Web information to generate utterances. In the proposed system, 
the conversation character can behave more naturally toward the user and talk flexibly. In the future, we intend to develop this system and obtain natural-speaking characters that can engage in interesting conversations.

\section{References}

1. Andre, E., Binsted, K., Tanaka-Ishii, K., Luke, S., Herzog, G., Rist, T.: "Three RoboCup simulation league commentator systems. Artificial Intelligence Magazine (21), 73-85 (Spring 2000)

2. Akiyo, N., Taeko, H., Hiroyuki, K., Ikuo, S., Katsumi, T.: Automatic Conversion and a Scripting Markup Language for Passive Watching and Listening of the Web Contents. Transactions of Information Processing Society of Japan 42(SIG1) (TOD8), 103-116 (2001)

3. Akiyo, N., Katsumi, T.: Passive Viewing of Web Page based on Automatic Daialog Generation. IPSJ SIG Notes 2004(72) 2004-DBS -134(I), 183-190 (2004); Cavazza, M., Charles, F., Mead, S.J.: AI-based Animation for Interactive Storytelling. In: Proceedings of Computer Animation, pp. 113-120. IEEE Computer Society Press, Seoul (2001)

4. Hourai, H., Akiyo, N., Katsumi, T.: Transformation from Web contents into humorous userfriendly talk show type contents. DBSJ Letters 2(2), 29-32 (2003)

5. Kitamura, Y., Yamada, T., Kokubo, T., Mawarimichi, Y., Yamamoto, T., Ishida, T.: Interactive integration of information agents on the web. In: Klusch, M., Zambonelli, F. (eds.) CIA 2001. LNCS, vol. 2182, pp. 1-13. Springer, Heidelberg (2001)

6. Kitamura, Y., Sakamoto, T., Tatsumi, S.: A competitive information recommendation system and its behavior. In: Klusch, M., Ossowski, S., Shehory, O. (eds.) CIA 2002. LNCS, vol. 2446, pp. 138-151. Springer, Heidelberg (2002)

7. Mehrabian, A.: Communication without words. Psychological Today 2, 53-55 (1968) 